\title{
Injection of aluminum potassium sulfate and tannic acid in the treatment of fecal incontinence: a single-center observational study
}

Tatsuya Abe, Masao Kunimoto, Yoshikazu Hachiro, Kei Ohara, Mitsuhiro Inagaki

Department of Proctology, Kunimoto Hospital, Asahikawa, Hokkaido, Japan

\section{Graphic Abstract}

\section{Purpose To evaluate the effects of injection therapy using aluminum potassium sulfate and tannic acid (ALTA) in the management of fecal incontinence (FI)}

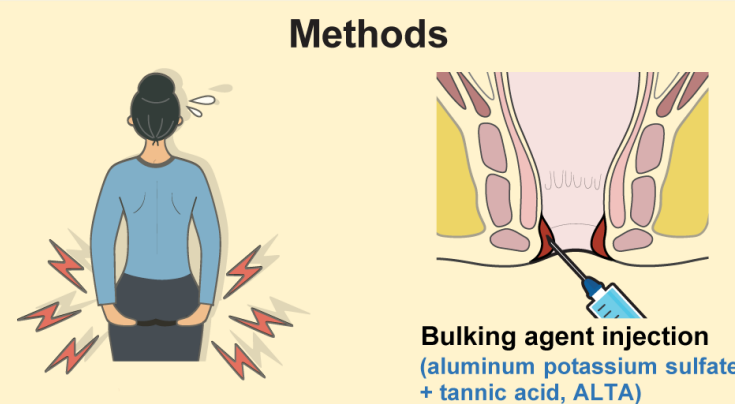

$\mathrm{N}=77$

2009-2019

Failed conventional treatment for $\mathrm{F}$

Exclusion

- concomitant large rectocele $(>4 \mathrm{~cm})$

- full-thickness rectal prolapse

- Combined hemorrhoidectomy with ALTA

Injection site:

3 main hemorrhoids and a

multipoint injection within anal
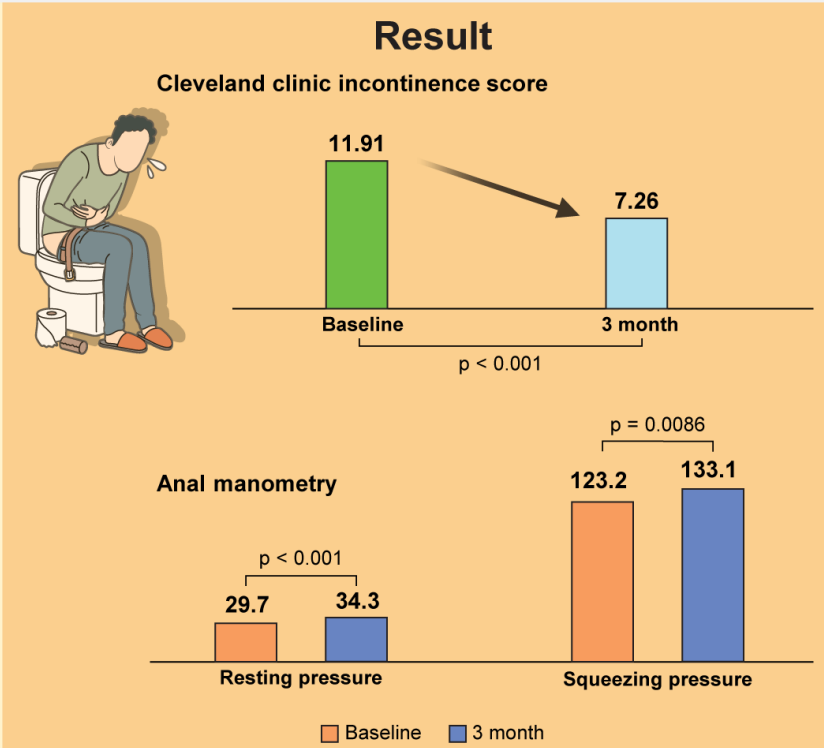

$\square$ Baseline $\square 3$ month
Conclusion Aluminum potassium sulfate and tannic acid (ALTA) injection as an injectable bulking agent is safe and a promising alterative for the treatment of fecal incontinence.

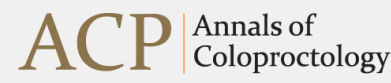

Abe et al.
Received: Apr 5, 2021 - Revised: May 4, 2021 - Accepted: May 7, 2021 Correspondence to: Tatsuya Abe, M.D., Ph.D.

Department of Proctology, Kunimoto Hospital, 4-5-1, Asahikawa, Hokkaido 070-0034, Japan

Tel: +81-166-25-2241, Fax: +81-166-23-1726

E-mail: t-abe@cf6.so-net.ne.jp

ORCID: https://orcid.org/0000-0002-7215-3597
() 2022 The Korean Society of Coloproctology

This is an open-access article distributed under the terms of the Creative Commons Attribution NonCommercial License (https://creativecommons.org/licenses/by-nc/4.0) which permits unrestricted noncommercial use, distribution, and reproduction in any medium, provided the original work is properly cited. 
Purpose: Perianal injection of bulking agents is an attractive treatment option for patients with mild to moderate fecal incontinence (FI). Various bulking agents have been used for injection therapy, but the optimal injection materials and methods are yet to be standardized. This study aimed to evaluate the effects of injection therapy using aluminum potassium sulfate and tannic acid (ALTA) in the management of FI.

Methods: This study included consecutive patients who underwent ALTA injection therapy for FI at our institution. The procedure was performed with the patient in the jackknife position, under caudal epidural anesthesia. The procedure consisted of a 4-step injection to the 3 main cushions and a multipoint injection to the remaining submucosa of the anal canal. Results: Seventy-seven patients (mean age, 76 years) were enrolled in the study. The mean Cleveland Clinic incontinence score of $11.9 \pm 4.1$ at baseline significantly improved to $7.3 \pm 5.2$ at 3 months following treatment. The mean maximal resting pressure also increased significantly 3 months after the intervention. Postoperative complications were observed in 3 patients (3.9\%), and all events were mild. The mean duration of postoperative follow-up was 17.5 months. The cumulative recurrence-free rate at 3 years was $72.4 \%$.

Conclusion: ALTA injection for FI is safe, easy to perform, and provides reasonable mid-term outcomes. Moreover, concomitant anorectal diseases that may be contraindicated by other injectable bulking agents could be treated simultaneously. Therefore, ALTA injection is a promising alternative in the absence of other injectable agents.

\section{Keywords: Fecal incontinence, Anal canal, Sclerotherapy, Aluminum potassium sulfate and tannic acid}

\section{INTRODUCTION}

Fecal incontinence (FI) is the involuntary loss of liquid or solid stool, which is a social or hygienic problem [1]. FI has a negative effect on the quality of life and may lead to severe social restrictions. The management of FI can be challenging owing to its multifactorial etiology. Conservative treatments such as dietary modifications, pharmacological treatment, and biofeedback are recommended as first-line therapies [2,3]. However, if conservative measures fail, surgical intervention may be required.

FI can be divided into 3 clinical subtypes; urge incontinence, passive incontinence, and mixed presentation [4]. Urge incontinence is suggestive of poor external anal sphincter (EAS) function, whereas passive incontinence is usually associated with internal anal sphincter (IAS) dysfunction [1]. The IAS has a greater impact on resting anal pressure. Endoanal sonography has enabled increased detection of FI secondary to IAS injury or degeneration [5]. IAS dysfunction is the most common cause of FI in Japan (35\%) [6]. Direct surgical repair of IAS defects is difficult, and the outcomes remain poor [7]. Further, the role of sacral neuromodulation in isolated IAS dysfunction has not yet been proven [8]. Therefore, the treatment modalities for IAS dysfunction are very limited.

Perianal injection of bulking agents is therefore an attractive alternative for patients with IAS dysfunction. Injectable bulking agents are intended to expand tissue in the anal canal and enhance resting anal pressures, thereby improving FI symptoms [2]. Various bulking agents have been utilized for injection therapy, but the optimal injection materials and methods have yet to be standardized [9]. In a recent Cochrane review of perianal bulking agents, the authors concluded that there is limited evidence to demonstrate the clinical benefit of silicone biomaterials and carboncoated beads, but dextranomer in hyaluronic acid (non-animal stabilized hyaluronic acid/dextranomer, NASHA Dx) gel improved FI in 52\% of patients, and these results were maintained during long-term follow-up [9-11]. NASHA Dx is a relatively new material that is injected into the submucosa of the anal canal, and it is currently the only product approved by the U.S. Food and Drug Administration (FDA) [2]. The Gatekeeper and Sphinkeeper (THD SpA, Correggio, Italy) are the latest self-expandable prostheses that can be implanted in the intersphincteric space of the anal canal and have produced favorable mid-term results $[12,13]$.

Although the use of perianal injections for treating FI is increasing in Europe and North America, no bulking agent has been approved in Japan thus far [14]. Injection sclerotherapy is an effective office procedure for treating hemorrhoids. Aluminum potassium sulfate and tannic acid (ALTA), an injectable sclerosant based on Xiaozhiling (Xiaozhiling zhusheye, Beijing, China) developed in China in the 1970s [15], has been used in Japan since 2005 [16]. ALTA injection proved to be highly effective even for grade III to IV hemorrhoids, and the effects were comparable with those of hemorrhoidectomy $[17,18]$. ALTA solution is not currently approved by the FDA; however, it is used in major Asian countries, including South Korea [19], and has also been applied in the treatment of rectal prolapse and rectocele [20-23].

The active ingredients of ALTA are alum and tannic acid. While alum instigates a strong local inflammatory reaction, tannic acid suppresses any excessive inflammation that may be caused by the alum and plays a critical role in alleviating any consequential tissue damage. Therefore, ALTA causes sclerosis and retraction of 
hemorrhoids through fibrosis associated with alum-induced chronic granulomatous inflammation [16]. In case of rectal prolapse or mucosal prolapse, ALTA is also injected into the region without hemorrhoidal cushions [20, 21]. Hence, persistent fibrosis and sclerosis develop circumferentially in the submucosal layer, which may lead to thickening and toughening of the anal canal and rectal wall. Furthermore, we previously reported that when ALTA injection was administered in patients with low resting anal pressure, the maximal resting pressure (MRP) significantly increased following treatment [24]. Therefore, we believe that ALTA injection has a similar mechanism to injectable bulking treatment.

Accordingly, we hypothesized that ALTA injection would be as effective as an injectable bulking agent for the treatment of FI. This study aimed to examine whether ALTA injection could be an alternative to injectable bulking treatment.

\section{METHODS}

This retrospective observational study analyzed the efficacy and safety of ALTA injections for FI. The study was approved by the Institutional Review Board of Kunimoto Hospital (No. K09-002), and written informed consent was obtained from all the patients.

\section{Study population}

We included consecutive patients who visited our hospital and underwent ALTA injection therapy for FI between January 2009 and December 2019. All the patients had a history of failed standard conventional treatments for FI, including antidiarrheal agents, anal electrical stimulation, and biofeedback. Patients with urge incontinence with significant EAS defects were excluded from the study. ALTA injection therapy has been approved only for internal hemorrhoids by the Japanese Health Insurance [16]. Therefore, FI patients with internal hemorrhoids (up to grade III) were selected as candidates and were first treated with topical ointments concurrent with conventional treatment for FI. Patients whose FI symptoms persisted even after the conventional treatment were included in the study. Patients who received combination therapy with hemorrhoidectomy and ALTA injection due to hemorrhoid progression despite topical ointment therapy were excluded. Patients with concomitant large rectocele $(>4 \mathrm{~cm})$ or grade $\mathrm{V}$ intussusception (full-thickness rectal prolapse) [25] were also excluded from the study because those symptoms may obscure the FI symptoms.

\section{Clinical assessment}

Preoperative assessment included a questionnaire regarding FI, Cleveland Clinic incontinence score (CCIS) [26], digital rectal examination, and anoscopy, followed by anal manometry, endoanal sonography, and barium defecography, if required. The CCIS was the sum of 5 parameters scored on a scale of 0 (absent) to 4 (daily). A total score of 0 suggested full continence and a score of 20 indi- cated complete FI [26].

Anal manometry was performed using a $5-\mathrm{mm}$ diameter, 1-channel, solid-state catheter with the patient in the left lateral position. The MRP was recorded using a rapid pull-through technique and was defined as the highest recorded resting pressure. Next, the maximal squeeze pressure (MSP), defined as the highest recorded pressure above the baseline (zero) at any level within the anal canal, was measured. Subsequently, the IAS and EAS were scanned by endoanal sonography using a 7-MHz rotating probe.

\section{Treatment procedure}

ALTA injection was administered in the operating room under caudal epidural anesthesia with the patients in the jackknife position. In patients taking anticoagulants, local anesthesia was administered to avoid spinal epidural hematoma. The ALTA injection method consisted of a 4-step injection for the 3 main hemorrhoids and a multipoint injection for the other area within the anal canal. Using a Z-type proctoscope (Arakawa Seisakujo, Tokyo, Japan), ALTA solution (Zione, J-Dolph Corp., Osaka, Japan) was slowly injected into 4 parts of each internal pile; submucosa of the superior pole, submucosa and mucosal layer of the central pile, and submucosa of the inferior pole (4-step injection) [17]. For small hemorrhoids, injection to the superior pole or inferior pole was omitted. Next, ALTA $(0.5-1.0 \mathrm{~mL})$ was evenly injected into the remaining submucosa of the anal canal at 5 to 10 locations (multipoint injection) [20, 21]. In cases with coexisting rectal intussusception or rectocele, a multipoint injection was performed on the corresponding rectal submucosa. The injected tissues were gently kneaded to evenly distribute the ALTA solution for each injection. Postoperatively, the patients who underwent caudal epidural anesthesia were hospitalized overnight.

\section{Outcome measures}

The patients were followed up at 2 weeks, then at 1,3 , and 6 months, and at 1, 2, and 3 years after ALTA injection. Subsequent follow-ups were voluntary. The follow-up comprised a questionnaire based on the CCIS, digital rectal examination, anoscopy, and anal manometry. Recurrence was defined as a re-increase in CCIS to a level at or above the baseline, or cases requiring additional operative interventions for FI. All data were retrospectively collected from the medical records. The CCIS, MRP, and MSP at baseline and 3 months after treatment were compared. The outcome measures were changes in CCIS, MRP, and MSP, postoperative complications, and cumulative recurrence rates.

\section{Statistical analysis}

Categorical variables are reported as frequencies and percentages, whereas continuous variables are reported as means and standard deviations (SD). The Wilcoxon signed-rank test was used to comparatively analyze the CCIS, MRP, and MSP values. The results are presented as means \pm SD values. The 2 -sided significance level was set at $5 \%$. The cumulative recurrence-free rate was assessed 
using the Kaplan-Meier method. All statistical analyses were performed using EZR software ver. 1.11 (Saitama Medical Center, Jichi Medical University, Saitama, Japan).

\section{RESULTS}

From January 2009 to December 2019, ALTA injection therapies were performed in overall 6,236 patients at our hospital. Of these, while 4,727 patients (75.8\%) underwent combination therapy with ALTA injection and hemorrhoidectomy, 1,509 (24.2\%) underwent ALTA injection therapy alone. Of these 1,509 patients, 77 (5.1\%) (63 females; mean age, 76.4 years) met the inclusion criteria and were included in the study. Patient characteristics at baseline are summarized in Table 1. ALTA injection was administered under caudal epidural anesthesia in 74 patients (96.1\%) and under local anesthesia in 3 patients (3.9\%). The planned injection procedure was successful in all the patients. The mean operative time was $7.8 \pm 3.7$ minutes, and the mean dose of ALTA per patient was $21.2 \pm 6.4 \mathrm{~mL}$.

Table 1. Baseline patient characteristics $(n=77)$

\begin{tabular}{|c|c|}
\hline Variable & Value \\
\hline Age (yr) & $76.4 \pm 7.7$ \\
\hline Female sex & $63(81.8)$ \\
\hline Height (cm) & $150.3 \pm 7.8$ \\
\hline Weight (kg) & $50.7 \pm 8.1$ \\
\hline \multicolumn{2}{|l|}{ Vaginal delivery $(n=63)$} \\
\hline 0 & $6(9.5)$ \\
\hline $1-2$ & $34(54.0)$ \\
\hline$\geq 3$ & $23(36.5)$ \\
\hline \multicolumn{2}{|l|}{ Stool frequency } \\
\hline$<3$ times/wk & $12(15.6)$ \\
\hline$\geq 3$ times/wk to $\leq 3$ times/day & $54(70.1)$ \\
\hline$>3$ times/day & $11(14.3)$ \\
\hline \multicolumn{2}{|l|}{ Bristol stool form scale } \\
\hline Type 1, 2 & $17(22.1)$ \\
\hline Type 3-5 & $49(63.6)$ \\
\hline Type 6, 7 & $11(14.3)$ \\
\hline \multicolumn{2}{|l|}{ Clinical subtype } \\
\hline Urge incontinence & $2(2.6)$ \\
\hline Passive incontinence & $51(66.2)$ \\
\hline Mixed incontinence & $24(31.2)$ \\
\hline \multicolumn{2}{|l|}{ Coexisting anorectal disease } \\
\hline Hemorrhoids ( grade III) & $77(100)$ \\
\hline Intussusception ( grade IV) & $24(31.2)$ \\
\hline Rectocele ( $\leq 4 \mathrm{~cm}$ diameter) & $15(19.5)$ \\
\hline
\end{tabular}

Values are presented as mean \pm standard deviation or number (\%).
CCIS was analyzed in 69 patients who completed the questionnaire, and 57 patients $(82.6 \%)$ had a reduction in CCIS after treatment. Of these, $31(44.9 \%)$ had a $\geq 50 \%$ reduction in CCIS, and 7 (10.1\%) regained full continence (CCIS score of 0 ). The mean CCIS of $11.9 \pm 4.1$ at baseline significantly improved to $7.3 \pm 5.2,3$ months after treatment. All the CCIS sub-scores also significantly improved (Table 2). Changes in manometric data were analyzed in 71 patients who completed the tests. Both the mean MRP and MSP increased significantly 3 months after treatment (Table 3 ).

Postoperative complications were observed in 3 female patients

Table 2. Changes in the CCIS before and after treatment $(n=69)$

\begin{tabular}{lccc}
\hline Variable & Baseline & 3 Mo & P-value \\
\hline Total CCIS & $11.91 \pm 4.14$ & $7.26 \pm 5.18$ & $<0.001$ \\
Solid & $1.17 \pm 1.49$ & $0.68 \pm 1.33$ & 0.0193 \\
Liquid & $3.10 \pm 1.15$ & $1.44 \pm 1.56$ & $<0.001$ \\
Gas & $2.67 \pm 1.57$ & $2.06 \pm 1.72$ & 0.0059 \\
Wears pad & $3.05 \pm 1.51$ & $2.30 \pm 1.77$ & 0.0016 \\
Lifestyle alteration & $1.97 \pm 1.83$ & $1.24 \pm 1.76$ & $<0.001$ \\
\hline
\end{tabular}

Values are presented as mean \pm standard deviation.

CCIS, Cleveland Clinic incontinence score.

Table 3. Change in the manometric data before and after treatment $(\mathrm{n}=71)$

\begin{tabular}{lccc}
\hline Variable & Baseline & 3 Mo & P-value \\
\hline Maximal resting pressure $(\mathrm{mmHg})$ & $29.7 \pm 14.0$ & $34.3 \pm 16.1$ & $<0.001$ \\
Maximal squeeze pressure $(\mathrm{mmHg})$ & $123.2 \pm 71.3$ & $133.1 \pm 80.9$ & 0.0086 \\
\hline
\end{tabular}

Values are presented as mean \pm standard deviation.

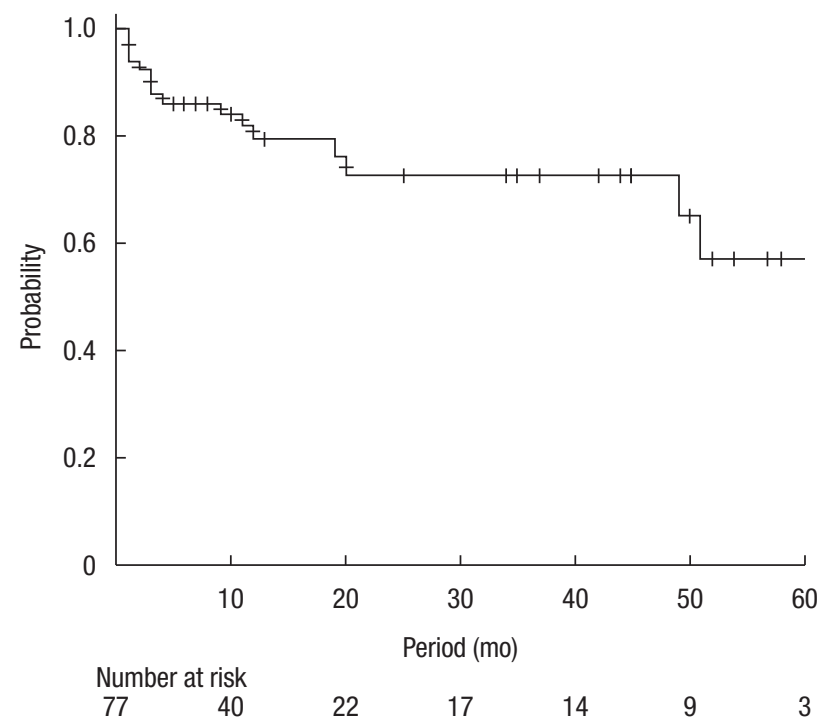

Fig. 1. Probability of no recurrence with time for patients who underwent the treatment. 
(3.9\%). One patient experienced fecal impaction, 1 had a fever of $\geq 38^{\circ} \mathrm{C}$, and the other patient had perianal dermatitis. All events were mild and ameliorated within days after treatment with sodium bicarbonate suppository, antipyretics, and topical ointments, respectively.

Of the 77 patients at baseline, 33 were available for follow-up after 12 months and 15 were continued follow-ups after 36 months. The mean duration of postoperative follow-up was 17.5 months (range, 1-84 months). Fig. 1 depicts the Kaplan-Meier plots of the recurrence-free probability for patients after treatment. Cumulative recurrence-free rates (95\% confidence interval) at 3 and 5 years were $72.4 \%(56.7 \%-83.3 \%)$ and $57.0 \%(33.1 \%-75.2 \%)$, respectively.

\section{DISCUSSION}

In this retrospective study of 77 patients, we demonstrated that ALTA injection for FI was safe, easy to perform, and achieved reasonable mid-term outcomes. This was confirmed by improvement in the validated severity index and objective manometric data.

The advantages of ALTA injection for FI are that it can be safely administered even to patients with concomitant anorectal diseases and those taking anticoagulants. ALTA injection has no risk of migration or dissipation like prostheses. Unlike other injectable bulking agents, repeated injections are not required. Furthermore, ALTA injection does not preclude future surgical options because there is no residual biocompatible material.

A Cochrane review of perianal bulking agents analyzed 5 randomized trials involving 382 patients [9]; most patients $(n=206)$ were derived from the recent placebo control trial by Graf et al. [10]. They reported that 71 of 136 patients (52.2\%) who received NASHA Dx had a $\geq 50 \%$ reduction in the number of incontinence episodes at 6 months [10]. In a review and meta-analysis of 23 articles (3 randomized controlled trials and 20 case series) in which 11 different bulking agents were used, 19 of the studies (637 patients) used the CCIS as a measure of therapeutic efficacy [27]. The pooled mean CCIS was 12.4 preoperatively and 7.7 at the last follow-up. The weighted mean difference in CCIS was 4.9, and the rate of improvement was $39.5 \%$ based on the CCIS [27]. In our study, $45 \%$ of patients exhibited a $\geq 50 \%$ reduction in CCIS and $10 \%$ achieved full continence; the rate of improvement was $38.7 \%$ based on CCIS. Therefore, we conclude that ALTA injection has a comparable effect on FI as other injection therapies.

In the above-mentioned review of 23 articles, 16 studies evaluated changes in manometric data after injection therapy. Mean MRP and MSP were the most commonly reported parameters [27]. In terms of change in MSP following injection, mixed results were observed, and no trend was noted. Meanwhile, 7 studies reported a significant increase in MRP during short-term follow-up [27]. Similarly, in our study, the mean MRP increased significantly after injection, whereas the MSP increased modestly.
The 23-article review involving 889 patients reported various adverse events [27]. The pooled rate of treatment-related adverse events was $18 \%$. They included perianal pain or discomfort (most common), leakage of injected agents, perianal abscess, mucosal erosion, obstructed defecation, hypersensitivity reaction, hematoma, and urgency [27]. The majority of adverse events were mild to moderate in intensity and resolved with nonsurgical management. However, in some cases, surgical drainage of the perianal abscess was required $[10,11]$. Our complication rate was only $4 \%$, and events were mild in all the patients. This confirms that ALTA injection for FI is an attractive option regarding safety.

Few studies have reported long-term outcomes evaluating durability of anal bulking agents $[9,28]$. Most studies suggest that the benefits of bulking agents appear to decline by 6 to 12 months [28]. Loss of treatment efficacy is likely related to the migration or dissipation of the bulking agents [9]. Meanwhile, the studies that evaluated the submucosal injection of NASHA Dx demonstrated that significant improvement in FI was sustained for up to 3 years $[10,11]$. In our study, the 3-year recurrence-free rate of ALTA injection for FI was as high as $72 \%$. We have vast experience in treating hemorrhoids with ALTA, and the long-term results were good $[16,18]$. Therefore, we expect that the long-term results of the ALTA injection for FI will also be favorable.

The limitations of this study include its retrospective, single-institution, and observational design without a control group. The observation period was shorter than that of studies of other injectable bulking agents. Another weakness is that no specific score was used to evaluate the quality of life before and after the treatment. While all patients had hemorrhoids, some had comorbidities such as rectal intussusception or rectocele, which can by themselves cause FI $[1,4]$. Consequently, our findings may have included the effects of correcting these comorbidities. Therefore, the results need to be confirmed by randomized controlled trials. Comparative studies with other injectable bulking agents are also required.

Patients with mild to moderate FI who experience mainly passive incontinence without significant EAS defects are suitable candidates for treatment with bulking agents $[4,9]$. Most of these patients are elderly and often have comorbidities, such as rectal mucosal prolapse or rectocele $[1,6]$. ALTA injection can simultaneously treat concomitant anorectal diseases that may be contraindicated by other injectable bulking agents. In conclusion, ALTA injection for FI is a promising alternative when current injectable bulking agents are not available.

\section{CONFLICT OF INTEREST}

No potential conflict of interest relevant to this article was reported.

\section{FUNDING}

None. 


\section{REFERENCES}

1. Maeda K, Yamana T, Takao Y, Mimura T, Katsuno H, Seki M, et al. Japanese Practice Guidelines for Fecal Incontinence Part 1-definition, epidemiology, etiology, pathophysiology and causes, risk factors, clinical evaluations, and symptomatic scores and QoL questionnaire for clinical evaluations-English version. J Anus Rectum Colon 2021;5:52-66.

2. Bharucha AE, Rao SS, Shin AS. Surgical interventions and the use of device-aided therapy for the treatment of fecal incontinence and defecatory disorders. Clin Gastroenterol Hepatol 2017;15: 1844-54.

3. Paquette IM, Varma MG, Kaiser AM, Steele SR, Rafferty JF. The American Society of Colon and Rectal Surgeons' clinical practice guideline for the treatment of fecal incontinence. Dis Colon Rectum 2015;58:623-36.

4. Wald A. Update on the management of fecal incontinence for the gastroenterologist. Gastroenterol Hepatol (N Y) 2016;12:155-64.

5. Vaizey CJ, Kamm MA, Bartram CI. Primary degeneration of the internal anal sphincter as a cause of passive faecal incontinence. Lancet 1997;349:612-5.

6. Mimura T, Yamana T, Takao Y, Seki M, Endo T, Katsuno H, et al. Current situation of the management of fecal incontinence in Japanese Institutions: diagnosis and treatment. J Jpn Soc Coloproctol 2012;65:101-8.

7. Morgan R, Patel B, Beynon J, Carr ND. Surgical management of anorectal incontinence due to internal anal sphincter deficiency. Br J Surg 1997;84:226-30.

8. Thaha MA, Abukar AA, Thin NN, Ramsanahie A, Knowles CH. Sacral nerve stimulation for faecal incontinence and constipation in adults. Cochrane Database Syst Rev 2015;(8):CD004464.

9. Maeda Y, Laurberg S, Norton C. Perianal injectable bulking agents as treatment for faecal incontinence in adults. Cochrane Database Syst Rev 2013;(2):CD007959.

10. Graf W, Mellgren A, Matzel KE, Hull T, Johansson C, Bernstein M, et al. Efficacy of dextranomer in stabilised hyaluronic acid for treatment of faecal incontinence: a randomised, sham-controlled trial. Lancet 2011;377:997-1003.

11. Mellgren A, Matzel KE, Pollack J, Hull T, Bernstein M, Graf W, et al. Long-term efficacy of NASHA Dx injection therapy for treatment of fecal incontinence. Neurogastroenterol Motil 2014;26: 1087-94.

12. Ratto C, Buntzen S, Aigner F, Altomare DF, Heydari A, Donisi L, et al. Multicentre observational study of the Gatekeeper for faecal incontinence. Br J Surg 2016;103:290-9.

13. La Torre M, Lisi G, Milito G, Campanelli M, Clementi I. Sphinkeeper $^{\mathrm{TM}}$ for faecal incontinence: a preliminary report. Colorectal Dis 2020;22:80-5.

14. Maeda K, Katsuno H, Tsunoda A, Seki M, Takao Y, Mimura T, et al. Japanese Practice Guidelines for Fecal Incontinence Part 3: surgical treatment for fecal incontinence, fecal incontinence in a special conditions: English version. J Anus Rectum Colon 2021;5: 84-99.

15. Shi Z, Zhou J, He X. On treatment of third-degree internal hemorrhoids with "Xiaozhiling" injection. J Trad Chin Med 1981;1:11520.

16. Yamana T. Japanese Practice Guidelines for Anal Disorders I. Hemorrhoids. J Anus Rectum Colon 2018;1:89-99.

17. Takano M, Iwadare J, Ohba H, Takamura H, Masuda Y, Matsuo K, et al. Sclerosing therapy of internal hemorrhoids with a novel sclerosing agent: comparison with ligation and excision. Int J Colorectal Dis 2006;21:44-51.

18. Hachiro Y, Kunimoto M, Abe T, Kitada M, Ebisawa Y. Aluminum potassium sulfate and tannic acid (ALTA) injection as the mainstay of treatment for internal hemorrhoids. Surg Today 2011;41: 806-9.

19. Lim SW. Aluminum potassium sulfate and tannic Acid injection for hemorrhoids. J Korean Soc Coloproctol 2012;28:73-7.

20. Hachiro Y, Kunimoto M, Abe T, Kitada M, Ebisawa Y. Aluminum potassium sulfate and tannic acid injection in the treatment of total rectal prolapse: early outcomes. Dis Colon Rectum 2007;50: 1996-2000.

21. Abe T, Hachiro Y, Kunimoto M. Combined aluminum potassium sulfate and tannic acid sclerosing therapy and anal encirclement using an elastic artificial ligament for rectal prolapse. Dis Colon Rectum 2014;57:653-7.

22. Abe T, Kunimoto M, Hachiro Y, Ebisawa Y. Injection sclerotherapy using aluminum potassium sulfate and tannic acid in the treatment of symptomatic rectocele: a prospective case series. Int J Surg 2016;30:94-8.

23. Kim JH, Lee YP, Suh KW. Changes in anorectal physiology following injection sclerotherapy using aluminum potassium sulfate and tannic acid versus transanal repair in patients with symptomatic rectocele; a retrospective cohort study. BMC Surg 2018;18:34.

24. Hachiro Y, Abe T, Kunimoto M, Satoh Y, Tsuruma T. Effect of ALTA therapy for internal hemorrhoids on anal functions. J Jpn Soc Coloproctol 2012;65:39-42.

25. Bordeianou LG, Carmichael JC, Paquette IM, Wexner S, Hull TL, Bernstein M, et al. Consensus Statement of definitions for anorectal physiology testing and pelvic floor terminology (Revised). Dis Colon Rectum 2018;61:421-7.

26. Jorge JM, Wexner SD. Etiology and management of fecal incontinence. Dis Colon Rectum 1993;36:77-97.

27. Hong KD, Kim JS, Ji WB, Um JW. Midterm outcomes of injectable bulking agents for fecal incontinence: a systematic review and meta-analysis. Tech Coloproctol 2017;21:203-10.

28. Koh CE, Solomon MJ. Injectable bulking agents for passive faecal incontinence. Tech Coloproctol 2013;17:343-4. 